\title{
Synchronous Activity in the Hippocampus and Nucleus Accumbens In Vivo
}

\author{
Yukiori Goto and Patricio O'Donnell \\ Center for Neuropharmacology and Neuroscience, Albany Medical College, Albany, New York 12208
}

The hippocampus is one of the brain regions involved in cognitive functions, including learning and memory. Extensive studies have unveiled how information is processed within this system. However, the mechanisms by which hippocampal activity is translated into action remain unsolved. One important target of hippocampal projections is the nucleus accumbens, which has been described as the motivation-to-action interface. Previous experiments indicate that these projections can control information processing in this region by setting neurons into a depolarized state. Here, we report that membrane potential transitions in nucleus accumbens neurons are correlated with electrical activity in the ventral hippocampus, suggesting that hippocampal neural activity can determine ensembles of active accumbens neurons.

Key words: nucleus accumbens; hippocampus; electrophysiology; schizophrenia; membrane potential states; in vivo intracellular recordings
The hippocampus, a brain region involved in cognitive functions (Zola-Morgan et al., 1986; Muller et al., 1996), projects extensively to the nucleus accumbens (NAcc) (Kelley and Domesick, 1982), a brain region at the "limbic-motor interface" (Mogenson et al., 1980). Previous experiments have suggested that these projections can control information processing in the NAcc by setting NAcc neurons into a depolarized state (O'Donnell and Grace, 1995). The electrical activity of NAcc and dorsal striatal medium spiny neurons is characterized by periodical shifts from a very negative resting membrane potential (DOWN state) to a more depolarized (UP) state (Wilson, 1993; O’Donnell and Grace, 1995), which depends on excitatory synaptic inputs (Wilson, 1993; O'Donnell and Grace, 1995). Transection or stimulation of the fimbria-fornix, the fiber system carrying hippocampal afferents to the NAcc, resulted in disappearance or induction, respectively, of the UP state in NAcc neurons (O'Donnell and Grace, 1995). Furthermore, recordings in slices do not yield neurons with a bistable membrane potential (O'Donnell and Grace, 1993). Because activation of inputs from the prefrontal cortex (PFC) can evoke action potentials in NAcc medium spiny neurons only during the UP membrane potential state, it has been hypothesized that hippocampal afferents gate PFC-NAcc information flow by setting NAcc neurons into this depolarized state. Such a gating mechanism may define the ensemble of neurons appropriate to be active in a given context (O'Donnell, 1999), and its alteration may be responsible for pathophysiological changes in psychiatric disorders, such as schizophrenia (O'Donnell and Grace, 1998).

A prediction that arises from this model is that electrical

Received Sept. 27, 2000; revised Dec. 4, 2000; accepted Dec. 6, 2000.

This work was supported by United States Public Health Service Grant MH57683. We thank Barbara L. Lewis for her excellent technical assistance, Drs. Stanley D. Glick and Min Zhou for comments on this manuscript, and Brian Lowry (University of Pittsburgh, Pittsburgh, PA) for developing and providing the software used for data acquisition and analysis (Neuroscope).

Correspondence should be addressed to Patricio O'Donnell, Albany Medical College (MC-136), Center for Neuropharmacology and Neuroscience, 47 New Scotland Avenue, Albany, NY 12208. E-mail: odonnep@mail.amc.edu.

Copyright (C) 2001 Society for Neuroscience $\quad 0270-6474 / 01 / 210001-\bullet \$ 15.00 / 0$ activity in the ventral hippocampus would be synchronized with UP states in NAcc neurons. For example, in hippocampal CA1 and ventral subicular (vSub) regions, local field potentials show periodical sharp potential shifts (sharp waves and accompanying high-frequency oscillation, or ripples) during sleep or awake resting conditions (Buzsáki, 1986; Ylinen et al., 1995). Sharp waves in the CA1 region are induced by synchronous discharge of CA3 neurons, resulting in depolarization of CA1 pyramidal neurons (Buzsáki, 1986). Therefore, outputs of such synchronous activity in the hippocampus may be the driving force of NAcc membrane potential fluctuations. To test this hypothesis, we performed in vivo intracellular recordings from NAcc medium spiny neurons simultaneously with local field potential recordings from the ventral hippocampus.

\section{MATERIALS AND METHODS}

Animals. Simultaneous in vivo intracellular and local field potential recordings were performed from 25 neurons in 19 rats. Male adult Sprague Dawley rats $(230-400 \mathrm{gm})$ were obtained from Taconic Farms (Germantown, NY). All experimental procedures were performed according to the United States Public Health Service Guide for the Care and Use of Laboratory Animals and approved by the Albany Medical College Institutional Animal Care and Use Committee. Rats were initially anesthetized with chloral hydrate $(400 \mathrm{mg} / \mathrm{kg}$, i.p.), followed by continuous supplemental anesthesia (chloral hydrate, $24-30 \mathrm{mg} / \mathrm{hr}$ ) during the recording session via a cannula inserted intraperitoneally.

Recordings. Intracellular and extracellular electrodes were made from $1 \mathrm{~mm}$ outer diameter Omegadot borosilicate glass tubing (World Preci-

This article is published in The Journal of Neuroscience, Rapid Communications Section, which publishes brief, peerreviewed papers online, not in print. Rapid Communications are posted online approximately one month earlier than they would appear if printed. They are listed in the Table of Contents of the next open issue of JNeurosci. Cite this article as: JNeurosci, 2001, 0:RC131 (1-5). The publication date is the date of posting online at www.jneurosci.org.

http://www.jneurosci.org/cgi/content/full/4996 
sion Instruments, Sarasota, FL) pulled with a P-97 Flaming-Brown puller (Sutter Instruments, Novato, CA). Intracellular electrodes (resistance of 46-103 M $\Omega$ ) were filled with $2 \mathrm{M}$ potassium acetate and 2\% Neurobiotin. Extracellular electrodes (impedance of 3-8 $\mathrm{M} \Omega$ measured at $2 \mathrm{kHz}$ ) were filled with $2 \mathrm{M} \mathrm{NaCl}$ and $2 \%$ Pontamine Sky blue. Intracellular electrodes were lowered into the NAcc [anteroposterior (AP), bregma +1.4 to $+2.0 \mathrm{~mm}$; lateral, $1.0-2.0 \mathrm{~mm}$; vertical, -5.8 to $-8.4 \mathrm{~mm}$ ] and extracellular electrodes into the ipsilateral CA1 (AP, bregma $-6.0 \mathrm{~mm}$; lateral, $5.3 \mathrm{~mm}$; vertical, $-7.8 \mathrm{~mm}$ ) or vSub (AP, bregma $-6.3 \mathrm{~mm}$; lateral, $5.2 \mathrm{~mm}$; vertical, $-7.0 \mathrm{~mm}$ ). Once intracellular electrodes yielded stable recordings, simultaneous baseline recordings were performed for 5 min. Only neurons showing at least $-50 \mathrm{mV}$ resting membrane potential and $40 \mathrm{mV}$ spike amplitude measured from threshold were analyzed and included in the study. All data were stored using custom-made software (Neuroscope) and analyzed off-line.

Histology. After completion of the experiments, extracellular recording sites were marked by ejection of Pontamine Sky blue. Neurobiotin was injected into the intracellularly recorded neurons by passing positive current $(1.0 \mathrm{nA}, 200 \mathrm{msec}$ pulses at $2 \mathrm{~Hz})$ for at least $5 \mathrm{~min}$. Animals were killed by a lethal dose of pentobarbital $(100 \mathrm{mg} / \mathrm{kg})$ and transcardially perfused with ice-cold saline, followed by $4 \%$ paraformaldehyde. Brains were removed from the skull, cryoprotected in $30 \%$ sucrose, and sectioned using a freezing microtome. Serial $50-\mu \mathrm{m}$-thick sections were cut coronally. Either dye markers or neurons were identified and localized according to the atlas of Paxinos and Watson (1998).

Cross-correlation analysis. Cross-correlograms were constructed based on the methods described previously (Perkel et al., 1967). The crosscorrelation strength, $S_{\text {ccr }}$, was defined as:

$$
S_{c c r}=\frac{N_{c}-N_{b}}{\sqrt{N_{m p} \times N_{l f p}}}
$$

where $N_{\mathrm{c}}$ is the number of potential shifts detected simultaneously in intracellular and extracellular recordings within a $\pm 50 \mathrm{msec}$ interval, $N_{\mathrm{b}}$ is the chance level of coincident events given by either shuffled crosscorrelogram or baseline events, and $N_{\mathrm{mp}}$ and $N_{\mathrm{lfp}}$ are the total number of events within the time intervals analyzed. Normal distribution was assumed for the histogram, and the significance of correlation was defined if the peak exceeded three SDs $(p<0.013)$.

\section{RESULTS}

In vivo intracellular recordings were performed from 25 NAcc neurons, located in the core region. Seventeen of $25(68 \%)$ neurons showed a bistable membrane potential (Fig. 1a). The presence of UP and DOWN membrane potential states was determined when a histogram of time spent at different membrane potential values showed a bimodal distribution fitting to a dual Gaussian curve (Fig. 1b). The DOWN state, or resting membrane potential (O'Donnell and Grace, 1995), was -78.1 \pm $10.5 \mathrm{mV}$ (mean $\pm \mathrm{SD}$ ), and the UP state was $-66.8 \pm 10.4 \mathrm{mV}$. Transitions to the UP state occurred at $0.55 \pm 0.21 \mathrm{~Hz}$. In all four bistable neurons injected with Neurobiotin, histochemical procedures revealed that these were medium spiny neurons (Fig. 1c).

Local field potentials in the hippocampus, either CA1 $(n=6)$ or vSub $(n=7)$, were recorded simultaneously with intracellular NAcc recordings from cells exhibiting a bistable membrane potential. These local field potentials exhibited large periodical negative shifts that occurred simultaneously with UP membrane potential states in NAcc neurons in all pairs recorded $(n=13)$ (Fig. 2a). Cross-correlograms indicated that hippocampal field potential shifts and transitions to the UP state in NAcc neurons occurred within $<100 \mathrm{msec}$ (Fig. $2 b$ ). Cross-correlation strength $\left(S_{\mathrm{ccr}}\right)$ was defined as the ratio between coincident number of events and total number of events. CA1-NAcc and vSub-NAcc recordings resulted in $S_{\mathrm{ccr}}$ of $0.27 \pm 0.08$ and $0.30 \pm 0.07$, respectively. These values were significantly higher than the strength of shuffled cross-correlograms $(0.05 \pm 0.01$ and $0.06 \pm$ 0.01 ; paired $t$ test; $p<0.00001$ for both of them). Because there was no difference in the strength and pattern of correlation

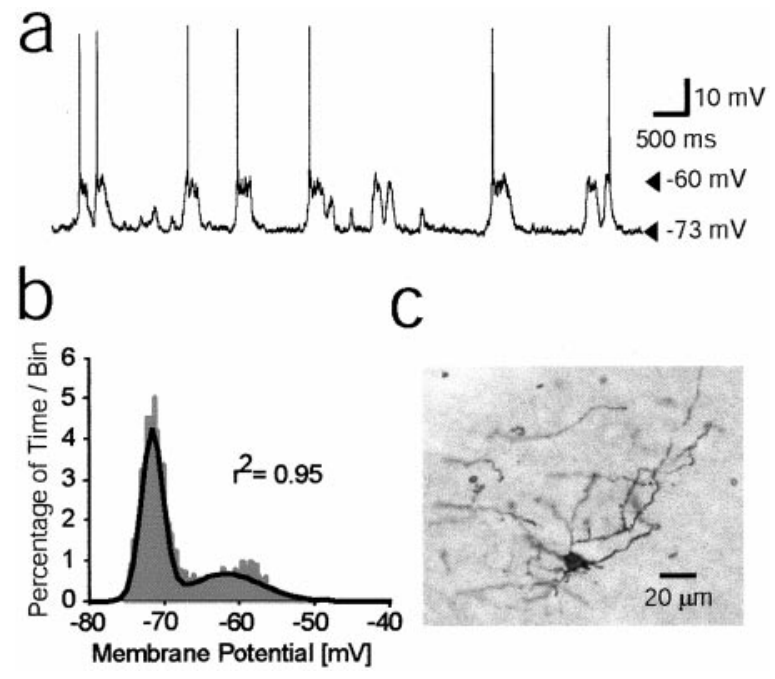

Figure 1. NAcc medium spiny neurons show a bistable membrane potential. $a$, Representative tracing from a neuron alternating between the DOWN $(-73 \mathrm{mV})$ and UP $(-60 \mathrm{mV})$ membrane potential states. $b$, Membrane potential distribution histogram constructed from the recording shown in $a$. Two peaks are observed, corresponding to the DOWN and UP membrane potential states. The black line is a dual Gaussian curve to which the histogram can be fitted $\left(r^{2}=0.95\right) . c$, Neurobiotin injection revealed that recordings were obtained from medium spiny neurons.

between NAcc and CA1 or vSub, the data were pooled. Half of the recordings obtained from the CA1 region exhibited ripples $(n=3)$, which were also synchronized with UP states in NAcc medium spiny neurons (Fig. 2c). Ripples were defined as oscillations of $>100 \mathrm{~Hz}$ (Buzsáki, 1986; Ylinen et al., 1995). An association was found between the location of NAcc neurons intracellularly recorded and their cross-correlation strength with hippocampal activity. $S_{\text {ccr }}$ values were higher for NAcc neurons located more medial and dorsal, in the medial core territory (Fig. $2 e$ ). Neurons located in the lateral core exhibited lower $S_{\mathrm{ccr}}$ with hippocampal activity. A statistically significant association was found between $S_{\text {ccr }}$ and the mediolateral location of the NAcc neurons (nonparametric Spearman rank order correlation; $r=$ $-0.554 ; p<0.05)$.

In addition to the hippocampal input, the NAcc receives excitatory afferents from the PFC (Phillipson and Griffiths, 1985). In a previous study, PFC stimulation failed to evoke transitions to the UP state in NAcc neurons (O'Donnell and Grace, 1995). It was then predicted that simultaneous recordings of intracellular NAcc activity and PFC local field potentials would yield a lower degree of cross-correlation compared with NAcc-hippocampus recordings. Although PFC local field potentials also exhibited slow negative potential shifts, they were not well correlated with transitions to the UP state in NAcc neurons $(n=4)$ (Fig. $3 a)$. Cross-correlograms showed significant peaks in only two of four pairs (Fig. 3b). Comparison of correlation strengths among PFCNAcc, shuffled PFC-NAcc, and hippocampus-NAcc pairs indicates that the cross-correlation between PFC local field potentials and NAcc membrane potential shifts was weaker than the crosscorrelation between hippocampal local field potentials and NAcc membrane potential transitions (ANOVA; $F_{(2,30)}=41.1 ; p<$ $\left.5.0 \times 10^{-7}\right)$ (Fig. 3c).

\section{DISCUSSION}

Hippocampal local field potentials were tightly correlated with transitions to the UP state in NAcc neurons, suggesting that 


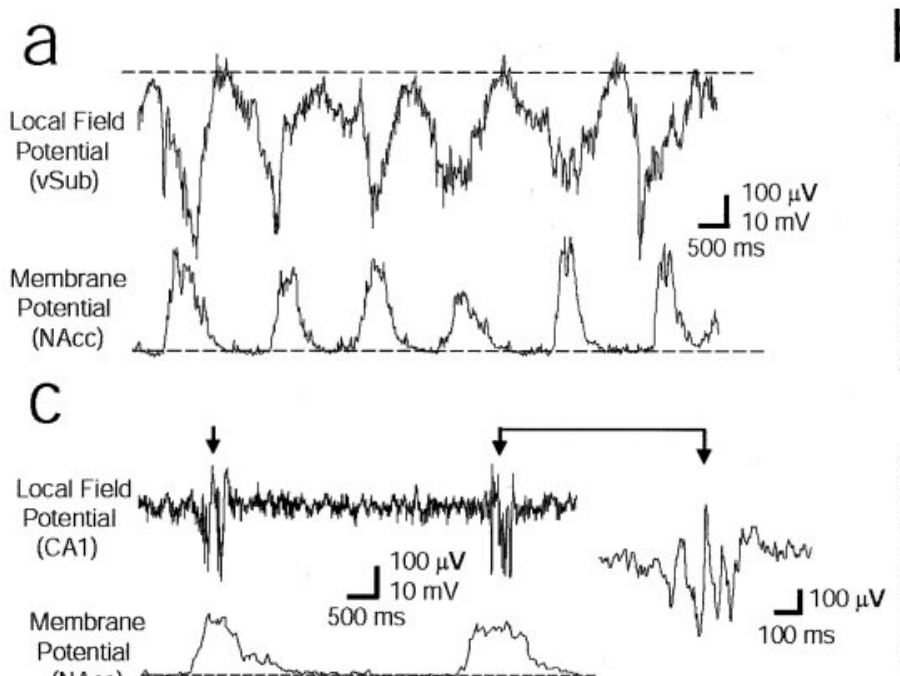

O

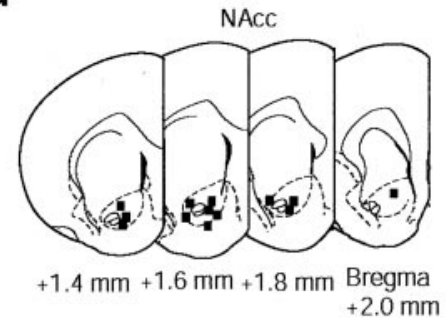

e
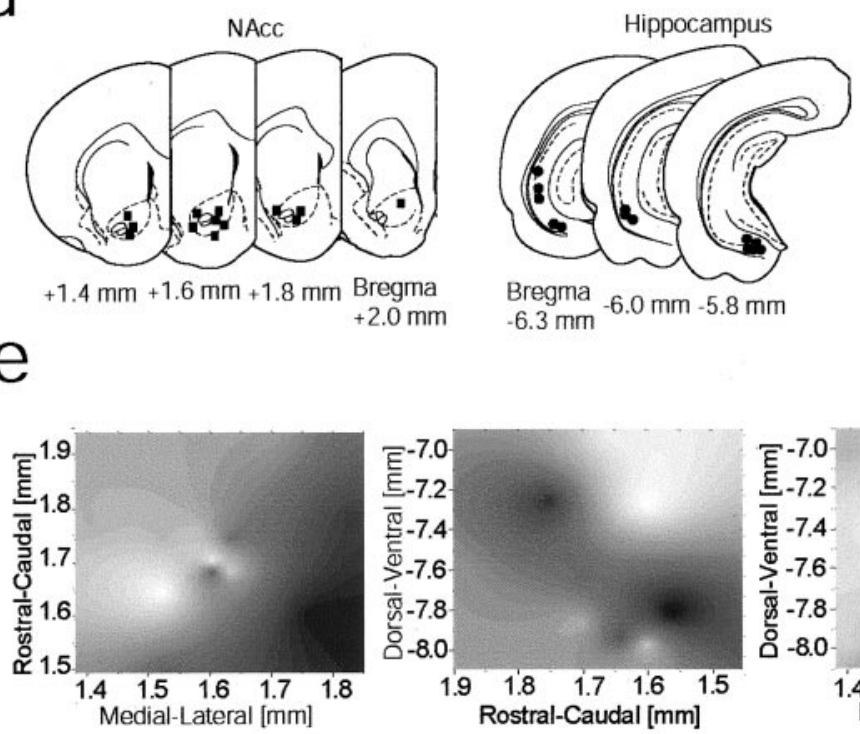

b
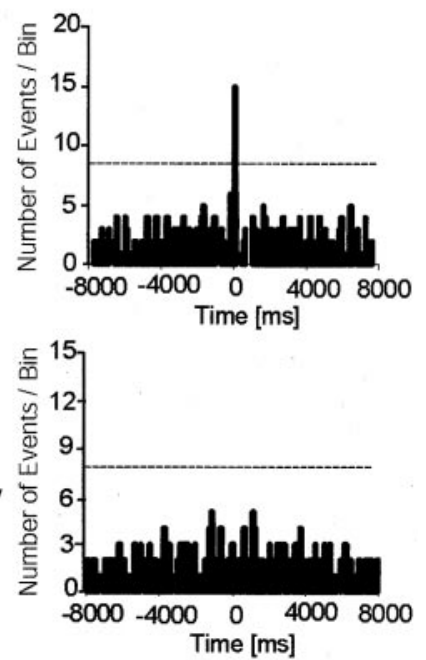

- Intracellular Recording

- Extracellular Recording

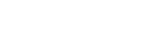

Figure 2. Membrane potential shifts in NAcc neurons occur simultaneously with hippocampal field potentials. $a$, Representative traces of simultaneous recordings from the NAcc and hippocampus. Top, Local field potential recorded from vSub showing periodical negative potential shifts; the dashed line indicates $0 \mu \mathrm{V}$. Bottom, Intracellular recording from an NAcc neuron showing a bistable membrane potential with UP events occurring synchronously with hippocampal field potential shifts; the dashed line indicates the DOWN state. Low-pass filter $(<50 \mathrm{~Hz})$ was applied to both traces to show only slow waveform components. $b$, Top, Cross-correlogram created from the recording shown in $a$. Bin width is 100 msec, and the dashed lines indicate three SDs. A significant peak is observed at time point 0 . Bottom, Shuffled cross-correlogram between nonsimultaneous time epochs of each recording does not reveal a peak. $c$, Example of simultaneous recordings indicating the correlation between hippocampal ripples recorded in CA1 and UP state transitions in an NAcc neuron. The inset shows one of the ripples at a faster time scale. High-pass filter $(>50 \mathrm{~Hz})$ was applied to the local field potential to eliminate slow potential shifts. $d$, Diagrams illustrating the locations of intracellular and local field potential recordings in the NAcc and hippocampus. $e$, The cross-correlation strength $\left(S_{\mathrm{ccr}}\right)$ between UP state transitions in an NAcc neuron, and onset of hippocampal local field potential shifts was plotted against the location of intracellularly recorded NAcc neurons on three two-dimensional grayscale maps. These maps were constructed from 13 pairs by plotting $S_{\text {ccr }}$ values in a $50 \times 50$ point matrix. The remaining $S_{\text {ccr }}$ values were determined by weighted least-squares estimation. Axes refer to coordinates in millimeters relative to bregma (Rostral-Caudal), midline (Medial-Lateral), and brain surface (Dorsal-Ventral).

hippocampal inputs are important for the onset of UP membrane potential states in NAcc neurons. In contrast, the correlation between PFC local field potentials and NAcc UP transitions was weak. Such difference between excitatory inputs to the NAcc is consistent with previous anatomical and physiological findings. Five to $10 \%$ of hippocampal terminals in the NAcc contact cell bodies or proximal dendrites, whereas PFC axons are exclusively observed at distal dendrites (Meredith et al., 1990). This anatomical arrangement would result in hippocampal afferents having a stronger input on somatic membrane potential in NAcc neurons.
Indeed, our finding of a cross-correlation strength between NAcc neurons and hippocampal local field potentials higher than that of NAcc-PFC pairs indicate that the PFC-NAcc projection is less important in driving the UP state than the hippocampal projections. Furthermore, the NAcc-hippocampal cross-correlation strength was higher when NAcc neurons were recorded from the medial aspect of the NAcc core. This may reflect the ventral hippocampus projecting primarily to the medial NAcc (Groenewegen et al., 1999).

UP and DOWN membrane potential states can be affected by 

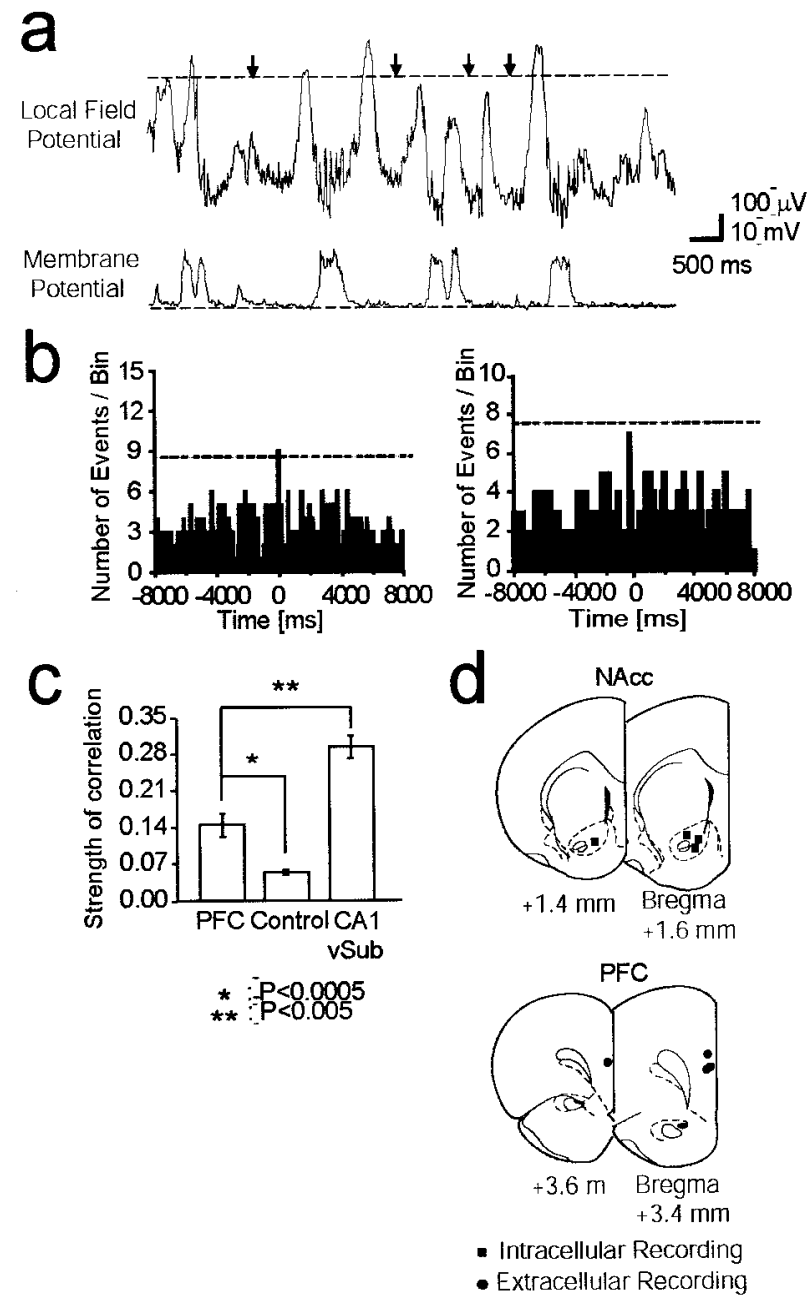

Figure 3. Membrane potential state transitions in NAcc neurons are correlated more weakly with prefrontal cortical local field potentials. $a$, PFC local field potential (top) acquired simultaneously with an intracellular recording from an NAcc neuron (bottom). The dashed lines indicate $0 \mu \mathrm{V}$ and the DOWN state in extracellular and intracellular recordings, respectively. Black arrows indicate PFC potential shifts that are not simultaneous with membrane potential transitions in the NAcc neuron. $b$, Cross-correlograms showed significant peaks in some cases (left); in others, the peak did not reach significant values (right). $c$, Comparison of cross-correlation strength among NAcc-PFC, control (shuffled NAccPFC), and NAcc-hippocampus pairs of recordings. A post hoc NewmanKeuls test was used for the comparison between each pair. $d$, Locations of intracellular recordings in the NAcc and local field potential recordings in the PFC.

the anesthetic agent used. Because the UP state is dependent on excitatory synaptic inputs, it cannot be seen in the presence of GABA-enhancing agents, such as barbiturates. On the other hand, these plateau depolarizations have been observed in chloral hydrate- or urethane-anesthetized animals (Wilson, 1993; O'Donnell and Grace, 1995) and even in locally anesthetized animals (Wilson and Groves, 1981).

Increasing attention is being paid to the role of neuronal ensembles in CNS information processing. Synchronous activity defining neural ensembles has been observed in the striatum (Jog et al., 1999), cerebellum (Thier et al., 2000), motor cortex (Laubach et al., 2000), PFC (Schoenbaum and Eichenbaum, 1995), and hippocampus (Deadwyler et al., 1996). These studies focused on spike firing in a population of simultaneously recorded
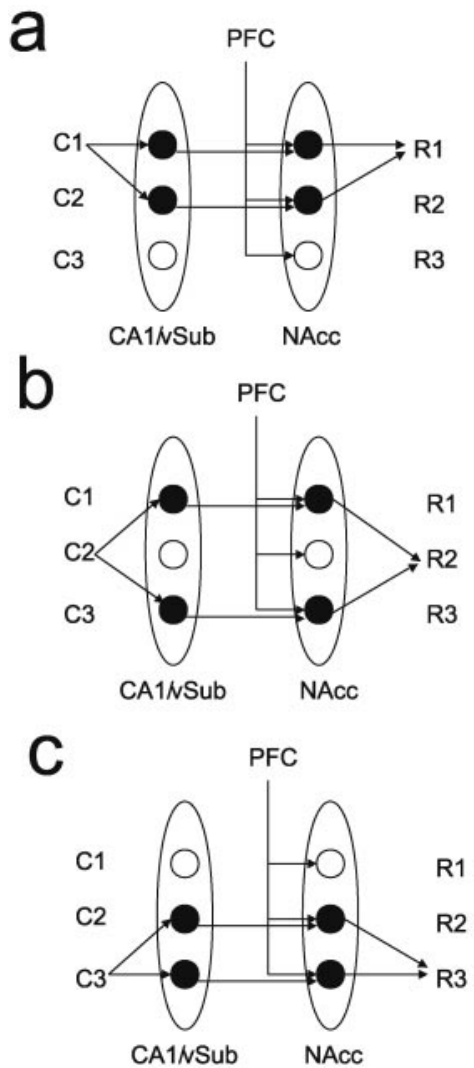

Figure 4. Hypothetical model of hippocampal gating of PFC-NAcc information transmission. Each circle indicates subsets of neurons. Different contextual information $(C 1-C 3)$ activates specific ensembles of ventral hippocampal neurons, which, in turn, shape ensembles of NAcc neurons defined as those in the UP state. Ensembles of active neurons in hippocampus (e.g., place fields) should be directly reflected on ensembles of NAcc neurons shaped by synchronous UP states because of parallel projections from the subiculum (Naber et al., 1997). Inputs from the PFC converging with the hippocampal-driven UP state in NAcc neurons can elicit specific responses $(R 1-R 3)$ according to such contextual information from the hippocampus.

neurons in behaving animals. Subthreshold membrane potential variations, however, were not considered. Because the UP membrane potential state brings NAcc neurons very close to their firing threshold, UP events can be seen as periods during which neurons are in a "ready-to-fire" state. Therefore, it would be important to consider the membrane potential states in the definition of active neuronal ensembles in systems exhibiting these oscillations (O’Donnell, 1999). Indeed, synchronous bistable membrane potential fluctuations in pairs of neurons have been reported recently in the dorsal striatum (Stern et al., 1998) and visual cortex (Lampl et al., 1999).

The results presented here support the idea of the hippocampal output gating corticostriatal information flow in the NAcc. The synchrony reported here is likely to be the result of hippocampal activity driving NAcc UP membrane potential states (O'Donnell and Grace, 1995). Thus, the activity of hippocampal neural ensembles may determine the ensembles of NAcc neurons that are appropriate to be set in the UP state, according to contextual cues (Fig. 4). This mechanism may provide a basis for the incorporation of hippocampal functions into motor planning and attentional mechanisms within the ventral basal ganglia circuits. 


\section{REFERENCES}

Buzsáki G (1986) Hippocampal sharp waves: their origin and significance. Brain Res 398:242-252.

Deadwyler SA, Bunn T, Hampson RE (1996) Hippocampal ensemble activity during spatial delayed-nonmatch-to-sample performance in rats. J Neurosci 16:354-372.

Groenewegen HJ, Beijer AVJ, Wright CI, Lopes Da Silva PH, Pennartz CMA (1999) Hippocampal and amygdaloid interactions in the nucleus accumbens. Psychobiology 27:149-164.

Jog MS, Kubota Y, Connolly CI, Hillegaart V, Graybiel AM (1999) Building neural representations of habits. Science 286:1745-1749.

Kelley AE, Domesick VB (1982) The distribution of the projection from the hippocampal formation to the nucleus accumbens in the rat: an anterograde- and retrograde-horseradish peroxidase study. Neuroscience 7:2321-2335.

Lampl I, Reichova I, Ferster D (1999) Synchronous membrane potential fluctuations in neurons of the cat visual cortex. Neuron 22:361-374.

Laubach M, Wessberg J, Nicolelis MA (2000) Cortical ensemble activity increasingly predicts behaviour outcomes during learning of a motor task. Nature 405:567-571.

Meredith GE, Wouterlood FG, Pattiselanno A (1990) Hippocampal fibers make synaptic contacts with glutamate decarboxylaseimmunoreactive neurons in the rat nucleus accumbens. Brain Res 513:329-334.

Mogenson GJ, Jones DL, Yim CY (1980) From motivation to action: functional interface between the limbic system and the motor system. Prog Neurobiol 14:69-97.

Muller RU, Stead M, Pach J (1996) The hippocampus as a cognitive graph. J Gen Physiol 107:663-694.

Naber PA, Caballero-Bleda M, Jorritsma-Byham B, Witter MP (1997) Parallel input to the hippocampal memory system through peri- and postrhinal cortices. NeuroReport 8:2617-2621.

O'Donnell P (1999) Ensemble coding in the nucleus accumbens. Psychobiology 27:187-197.

O’Donnell P, Grace AA (1993) Physiological and morphological prop- erties of accumbens core and shell neurons recorded in vitro. Synapse 13:135-160

O’Donnell P, Grace AA (1995) Synaptic interactions among excitatory afferents to nucleus accumbens neurons: hippocampal gating of prefrontal cortical input. J Neurosci 15:3622-3639.

O'Donnell P, Grace AA (1998) Dysfunctions in multiple interrelated systems as the neurobiological bases of schizophrenic symptom clusters. Schizophr Bull 24:267-283.

Paxinos G, Watson C (1998) The rat brain in stereotaxic coordinates, Ed 4. San Diego: Academic.

Perkel DH, Gerstein GL, Moore GP (1967) Neuronal spike trains and stochastic point processes. II. Simultaneous spike trains. Biophys J 7:419-440.

Phillipson OT, Griffiths AC (1985) The topographic order of inputs to nucleus accumbens in the rat. Neuroscience 16:275-296.

Schoenbaum G, Eichenbaum H (1995) Information coding in the rodent prefrontal cortex. II. Ensemble activity in orbitofrontal cortex. J Neurophysiol 74:751-762.

Stern EA, Jaeger D, Wilson CJ (1998) Membrane potential synchrony of simultaneously recorded striatal spiny neurons in vivo. Nature 394:475-478.

Thier P, Dicke PW, Haas R, Barash S (2000) Encoding of movement time by populations of cerebellar Purkinje cells. Nature 405:72-76.

Wilson CJ (1993) The generation of natural firing patterns in neostriatal neurons. Prog Brain Res 99:277-297.

Wilson CJ, Groves PM (1981) Spontaneous firing patterns of identified spiny neurons in the rat neostriatum. Brain Res 220:67-80.

Ylinen A, Bragin A, Nadasdy Z, Jando G, Szabo I, Sik A, Buzsaki G (1995) Sharp wave-associated high-frequency oscillation $(200 \mathrm{~Hz})$ in the intact hippocampus: network and intracellular mechanisms. J Neurosci 15:30-46.

Zola-Morgan S, Squire LR, Amaral DG (1986) Human amnesia and the medial temporal region: enduring memory impairment following a bilateral lesion limited to field CA1 of the hippocampus. J Neurosci 6:2950-2967. 